\title{
Analysis of the nonlinear behavior of adhesives in bonded assemblies- Comparison of TAST and Arcan tests
}

\author{
J.Y. Cognard ${ }^{\mathrm{a},{ }^{*}}$, R. Créac'hcadec ${ }^{\mathrm{a}}$, L. Sohier ${ }^{\mathrm{b}}$ and P. Davies ${ }^{\mathrm{c}}$
}

\author{
a Mechanics of Naval and Offshore Structures, ENSIETA, 2 rue Verny, 29806 Brest, France \\ ${ }^{b}$ Laboratoire d'Ingénierie Mécanique et Electrique, UBO, 29285 Brest Cedex, France \\ ${ }^{c}$ Materials and Structures Group, IFREMER Brest Centre, 29280 Plouzané, France \\ *: Corresponding author : Cognard J. Y., email address : jean-yves.cognard@ensieta.fr
}

\begin{abstract}
:
This paper describes a study in which the shear behavior of a structural epoxy adhesive has been measured using the standard thick adherend shear test (TAST) specimen and a modified Arcan test. A numerical study of the TAST test taking into account the nonlinear behavior of the adhesive and the finite deformations of the adhesive joint, shows that there is a localization of plastic zones close to the adhesive-substrate interface near the free edge of the adhesive. Experimental tests carried out with steel and aluminum substrates and with various adhesives also show that failure initiates in this region. These edge effects in the TAST fixture can lead to an incorrect analysis of the behavior of the adhesive (for instance, underestimation of the shear stress in the joint at failure), particularly when an adhesive failure mode is dominating. The modified Arcan fixture provides a more homogeneous stress state. A similar improvement of the TAST fixture is proposed.
\end{abstract}

Keywords: Epoxy/epoxides; Stress distribution; Mechanical properties of adhesives; Adhesive testing

\section{Introduction}

The marine industry uses adhesively bonded assemblies, involving both metal and composite structures, on a daily basis, but the prediction of the behavior of these bonded joints is still approximate [1, 2]. Adhesively bonded joints offer many advantages compared to metallic fasteners for the design of floating structures, notably weight gain and improved corrosion resistance, but a lack of confidence limits the current use of this technology. Moreover, bonding in a boatyard environment involves joining large parts, with variable adhesive thickness, simple preparation of surfaces and cure at a low temperature. In previous work, in order to analyze the behavior of adhesively bonded joints, a modified Arcan fixture $[3,4]$, which allows 
compression or tension to be combined with shear loads through radial loading, has been designed enabling the adhesives of interest to be characterized up to failure. It has been numerically shown that the use of supports with a beak close to the adhesive joint makes it possible to limit the edge effects, and that the local geometry of the joint near the edge is an important parameter [5]. For the epoxy resin Vanticoô Redux 420 (Vantico Ltd, Duxford, Cambridge, UK, www.vantico.com/adhesives) different experimental studies have been performed and the fracture envelope in the normal stress shear stress plane has been obtained. Compression was shown to increase the shear stress at failure significantly [6]. Various other aspects of the nonlinear behavior of this adhesive have been revealed. Cyclic and relaxation loads indicated significant rate effects [6]. Results for mixed assemblies (steel, aluminium, composite substrates) show similar adhesive behavior for the surface preparations, cure and test conditions applied [6]. Thus the experimental study of an adhesive joint with metallic substrates can provide useful information for modelling the behavior of bonded assemblies.

For marine applications, it is important to analyze the influence of the temperatures, and ageing in an appropriate environment (seawater exposure), on the nonlinear behavior of the adhesive. The proposed Arcan fixture described above makes it possible to carry out these tests, but the standardized TAST (Thick Adherend Shear Test) method [7] may be better adapted for such 
studies. For example, Tomblin et al. [8] used the TAST in an extensive study of the influence of temperature and humidity on properties of structural adhesives. The TAST was therefore examined. However, a comparison of the experimental results, for different loading rates, for these two shear tests (Arcan and TAST) showed differences in the non-linear behavior as will be detailed below. In order to analyze those differences, various numerical Finite Element simulations have been developed. A detailed study of the distribution of the stresses in the adhesive joint, for the TAST fixture, showed that the edge effects are very significant. A numerical study, taking into account the nonlinear behavior of the adhesive and the finite deformations of the adhesive joint, shows that there is a localization of the plastic zones close to the adhesive-substrate interface near the free edge of the adhesive. In tests on assemblies of steel and aluminium substrates with various adhesives failure was also observed to initiate here. These edge effects in the TAST fixture can lead to an incorrect analysis of the behavior of the adhesive (for instance, underestimation of the shear stress in the joint at failure), in particular whenever an adhesive failure mode is dominating. Moreover, the influence of the substrate material on stress distribution in the adhesive joint is presented below for the two test fixtures.

The experience gained during the design improvement of the Arcan assembly (limitation of the edge effects and control of the stress distribution in the adhesive joint) made it possible to propose a modification of the TAST fixture to give a more reliable analysis of the behavior of the adhesive. Moreover, a modular TAST fixture using small samples is proposed. This is well adapted to the study of the influence of marine ageing on adhesive properties as a large number of small samples can be removed from bonded plates having been exposed to ageing, and tested in shear. Tests are underway to complete this numerical study.

\section{Presentation of the modified Arcan fixture}

In this section the main points of the design of the experimental Arcan fixture will be presented briefly, in order to explain the results which are used in the following sections of this paper. In order to characterize the behavior of an adhesive it is important to use a specimen for which the adhesive joint has a geometry similar to that used for the industrial assemblies, i.e. a thickness of some tenths of millimeters here. Single lap-shear or double lap-shear specimens, are the most widely used tests, but generate strong stress gradients [5]. Therefore it is quite difficult to analyze such experiments. The standardized TAST method allows the full stress-strain curve to be obtained but only in shear [7]. Adhesive failure envelopes are expensive to obtain and depend on many manufacturing parameters so adhesive suppliers rarely provide them. In order to determine these values a variant of the Arcan fixture [4] has been designed; it allows the adhesive, in thin films, to be loaded under a wide range of combinations of tension/compression and shear loads, using standard test machines (figure 1). Two important parameters in this test are the geometry of the substrate (beak) and the geometry of the joint near the edge. Some experimental and numerical illustrations of this are shown below.

\subsection{Experimental results}

Two important points were studied when designing this assembly. First, the generation of a stress field in the adhesive joint which is as uniform as possible with a maximum stress in the centre of the joint, away from the edges. This is important in order to limit the influence of defects. Second, the system fixing the substrates on the supporting fixture has to be designed in order to prevent pre-loading of the adhesive joint. The aim was to find geometries making it possible to reduce the contribution of the singularities due to edge effects. It has been shown that the use of a beak with an angle in the range between $30^{\circ}$ and $45^{\circ}$ makes it possible to cancel out stresses around the perimeter at the mean plane of the adhesive [4-5]. It is important to note that the connecting radius and the dimensions of the edge have only a weak influence on the stress distribution. Other techniques have been described elsewhere which allow a reduction of stress singularities in bonded assemblies [9-10].

To preserve the simplicity associated with the Arcan fixture and taking into account the 
machining constraints, a specimen with a rectangular section $(65 \mathrm{~mm} \times 10 \mathrm{~mm}$ ) was chosen (figure 1). Figure 1c presents the geometry of the end of the joint of the adhesive which is obtained by cleaning before curing.

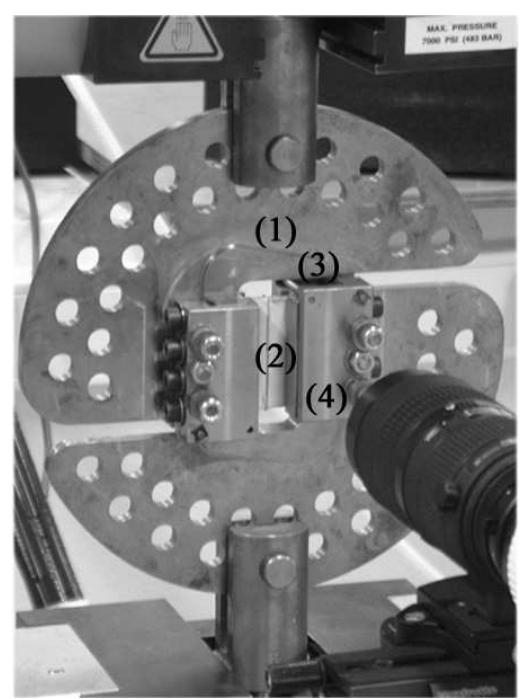

(a) Arcan fixture (shear loading)

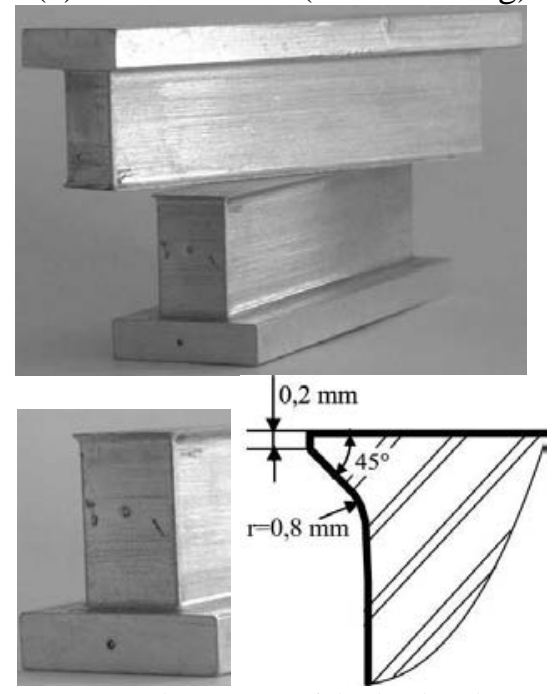

(c) substrates with the beak

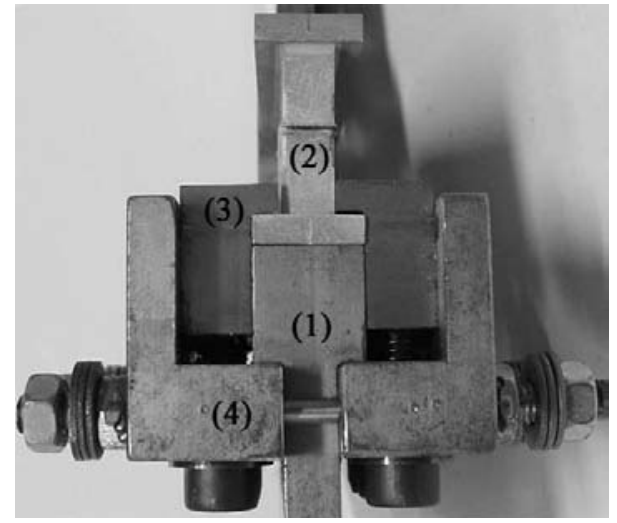

1 - support of the Arcan fixture

2 - bonded specimen

3 - clamping system

4 - support of the clamping system

(b) mounting of the specimen
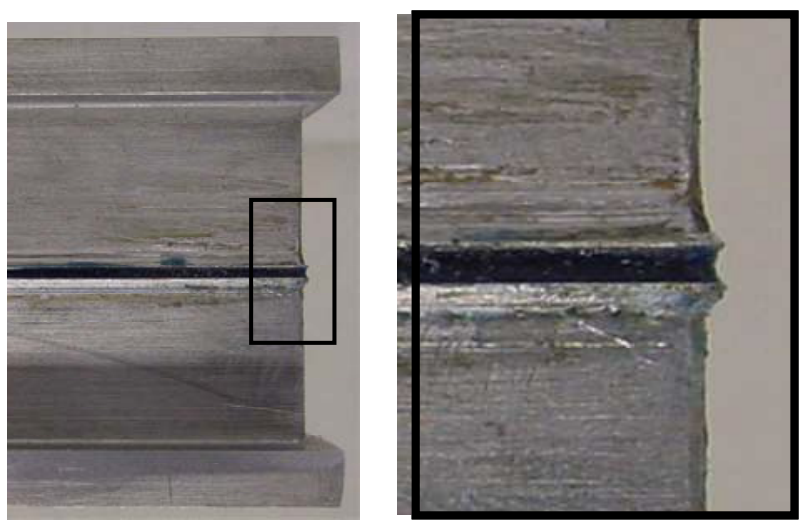

(d) geometry of the specimens

Fig. 1. Presentation of the modified Arcan fixture.

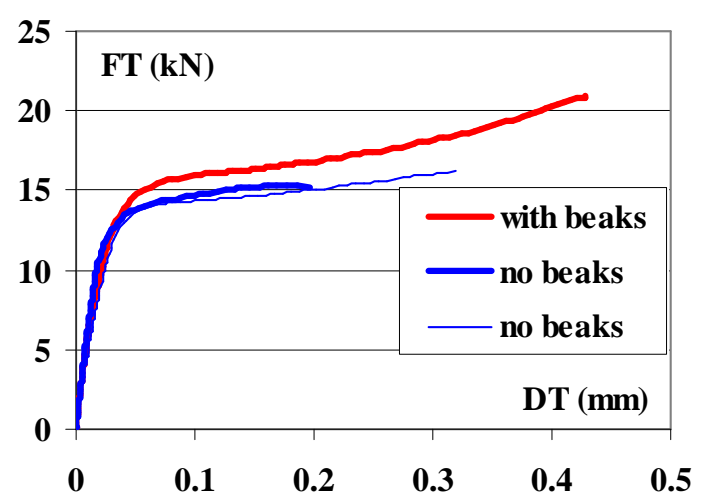

Fig. 2. Experimental results - shear loading.

Figure 2 shows some experimental results obtained with the modified Arcan fixture in the case of shear loading. For the results which follow, unless stated otherwise, the epoxy resin Vantico Redux 420 is used. It was left for 12 hours at $20^{\circ} \mathrm{C}$ after assembly on aluminium substrates then 
cured at $50^{\circ} \mathrm{C}$ for 4 hours; a thickness of the adhesive joint of $0.4 \mathrm{~mm}$, obtained using a special calibrated fixture [4] and an imposed displacement rate of $0.5 \mathrm{~mm} / \mathrm{minute}$ (crosshead of the tensile testing machine) were used. We denote by DT the relative displacements of both ends of the adhesive joint in the tangential direction in the mean plane of the adhesive joint; DT is obtained with a non-contact measurement system by image correlation [6]. FT represents the tangential component of the applied load in the normal to the mean plane of the adhesive joint (the normal component is equal to zero for shear loading).

It is important to note the significant differences between the results obtained with and without beaks (i. e. with straight substrates) in figure 2. Scatter was also larger when there were no beaks (figure 2).

\subsection{Numerical analysis}

Finite element analyses, assuming linear elastic behavior of the components, have been performed in order to determine the evolution of the stress through the thickness of the adhesive joint. As we have to model multi-material structures one must respect the mechanical properties of perfect interfaces. With the standard finite element method, based on the variational principle of minimum potential energy whose unique variable is the displacement field, the continuity of the displacement is satisfied but the continuity of the stress vector is not exactly verified. Therefore refined meshes are needed near the interface in order to obtain good numerical results, especially for large material heterogeneity of the assemblies [11-12]. Different numerical simulations, for an adhesive joint thickness of $0.4 \mathrm{~mm}$, have shown that good numerical results are obtained from meshes with 40 linear rectangular elements in the half-thickness (e) of the adhesive. Simulations with a larger number of elements (60 or more) showed no differences in results. Computations were made in 2D on half of the specimen by applying adequate boundary conditions: i.e. anti-symmetric condition along line Ox (figure 3). In order to obtain a realistic distribution of stresses within the adhesive joint near the edge, the real dimensions of the substrates were used $(\mathrm{OB}=15 \mathrm{~mm} ; \mathrm{OA}=32.5 \mathrm{~mm})$. The associated 2D finite element model uses non-uniform meshes in order to reduce the size of the linear problem to be solved. It uses 299710 degrees of freedom (around 146000 linear rectangular elements) to describe the adhesive and around 231000 degrees of freedom (around 227000 linear triangular elements) to describe a substrate. For the different computations under plane stress assumption, a displacement is applied to line CD. Results are presented for aluminium substrates (Young modulus $\mathrm{E}=80 \mathrm{GPa}$, Poisson's ratio $\mathrm{v}=0.3$ ). The material parameters for the adhesive are $\mathrm{E}=2.2 \mathrm{GPa}$ and $v=0.3$. Three different geometries were studied (figure 3): " $\mathrm{A}$ " a beak with a convex adhesive joint edge; "B" a beak with a straight edge of the adhesive joint; "C" a straight edge of the substrate with a straight edge of the adhesive joint. Results, Figure 4, have been normalized with respect to the value at the centre of the joint to make the analysis of curves presenting stress distributions easier. Figure $4 \mathrm{a}$ presents, for the three different geometries, the distribution of the stresses in the mean plane of the adhesive joint. In this figure the shear stress is identified as "SMXY". These results show that, in the mean plane of the adhesive, there is only shear stress for this experimental fixture under shear loading.

Figures $4 \mathrm{~b}-\mathrm{c}-\mathrm{d}$ present the evolution of the von Mises equivalent stress in the adhesive joint along different lines with respect to the position $\mathrm{y}$ in the adhesive joint: $\mathrm{y}=0$ corresponds to the midplane of the adhesive (segment OA, figure 3) and $\mathrm{y}=\mathrm{e}$ corresponds to the interface between adhesive and substrate. The shear stress is the main stress component, so a Mises equivalent stress is used, (though others could be applied). This is the most widely used approach for isotropic materials. Values are again normalized to " 1 " in the middle of the joint. Defects (manufacturing or loading) can increase the stress in the adhesive close to the edges; thus for geometry " $\mathrm{C}$ ", without beak, any defects, often found at the metal/adhesive interface, will lead to this interface being the most stressed part of the adhesive joint and this will affect the experimental results. These results show that the beak can limit the edge effects. The stress distribution in the middle part of the specimen does not depend on the geometry of the edge. The edge effects can be limited by decreasing the thickness of the end of the beak or by using a smaller angle to define the beak [5], 
but the beak is then mechanically weaker and prone to accidental damage. It is also interesting to examine numerically the edge effects when steel substrates are used instead of aluminium because the main parameter which governs the response is the ratio of the Young's modulus of the two materials (adhesive and substrate) [5]. Figure 5 presents some numerical results, for steel substrates, for geometry "A" $(\mathrm{E}=210 \mathrm{GPa}, \mathrm{v}=0.3)$. A comparison between the results presented in figures $4 \mathrm{~b}$ and $5 \mathrm{~b}$ shows that with the Arcan fixture the edge effects are more important for steel than for aluminium substrates. In the case of geometry "A", for aluminium substrates the maximum value of the normalized Mises equivalent stress close to the adhesive free edges is equal to nearly 0.4 and for steel substrates the corresponding value is nearly equal to 0.8 .

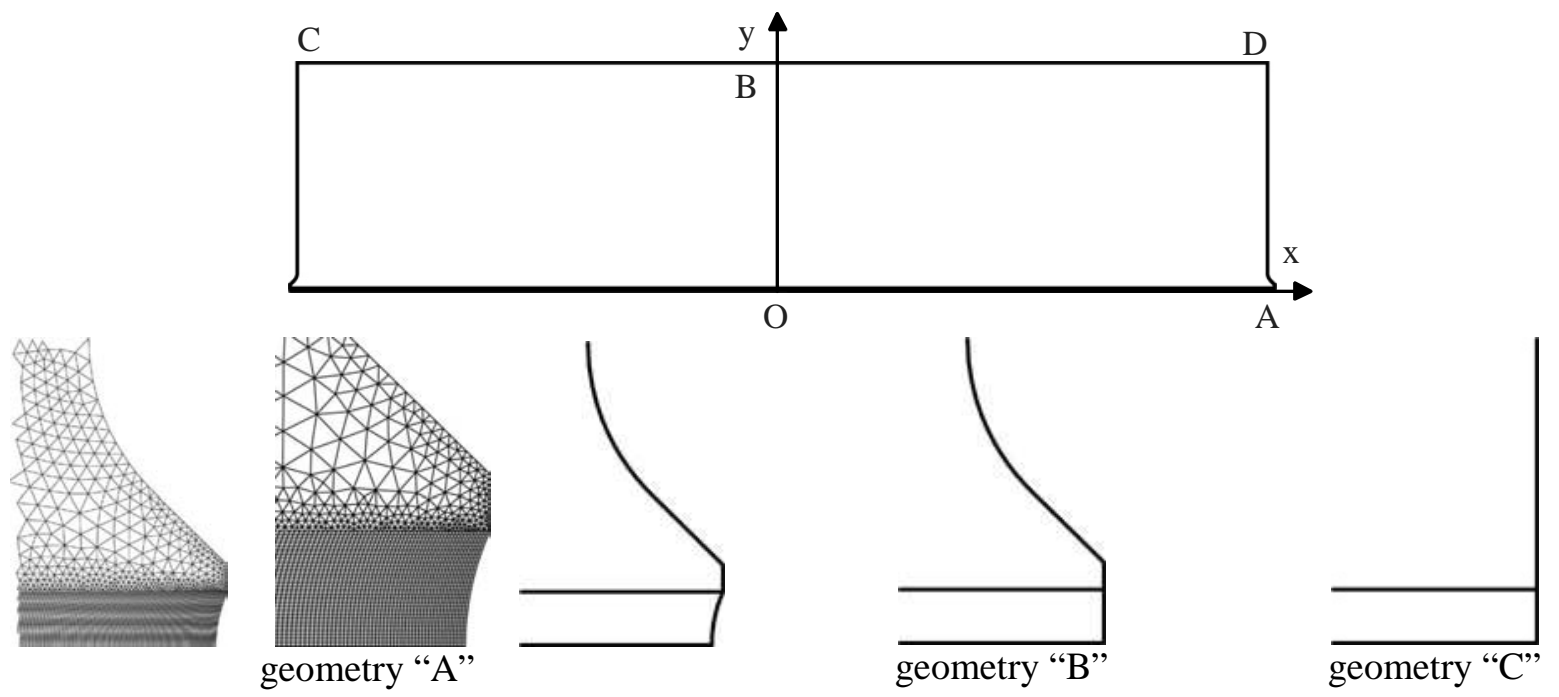

Fig. 3. Geometry used, mesh near the edge and different geometries near the edge of the joint.

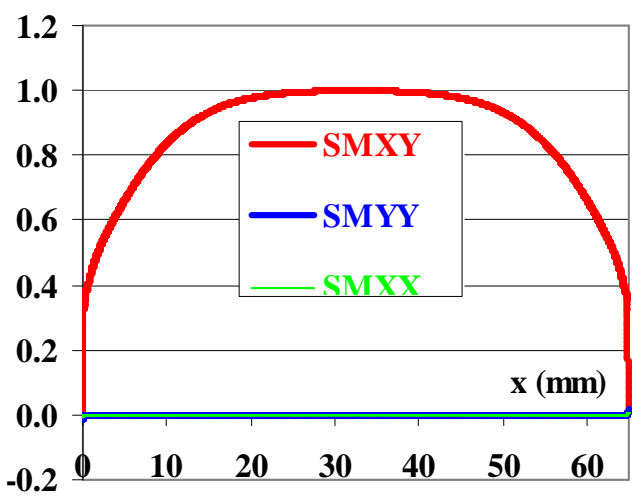

(a) stress distribution in the mean plane of the adhesive

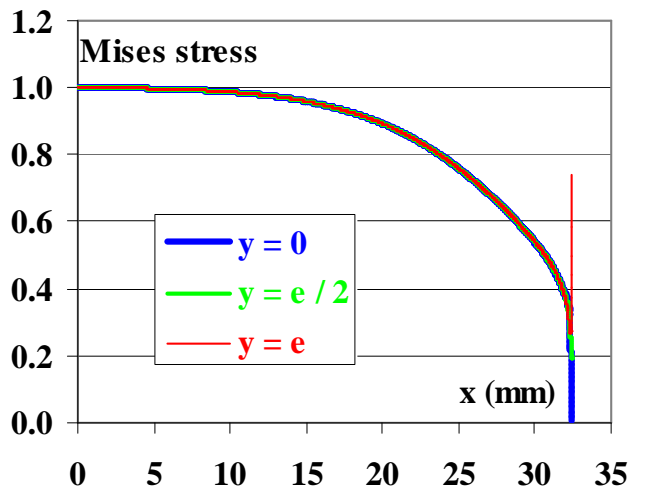

(c) results for geometry " $\mathrm{B}$ "

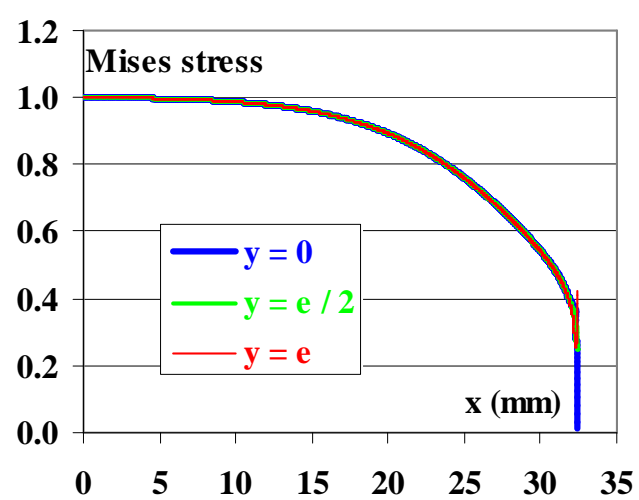

(b) results for geometry " $\mathrm{A}$ "

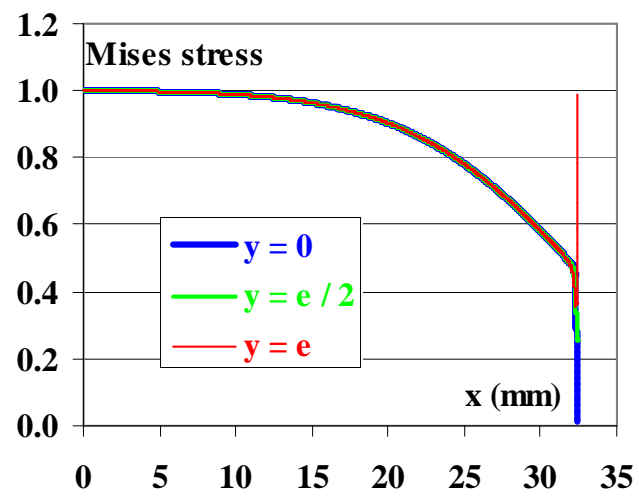

(d) results for geometry " $C$ "

Fig. 4. Normalized stress through the thickness of the joint for different geometries (aluminium substrates). 


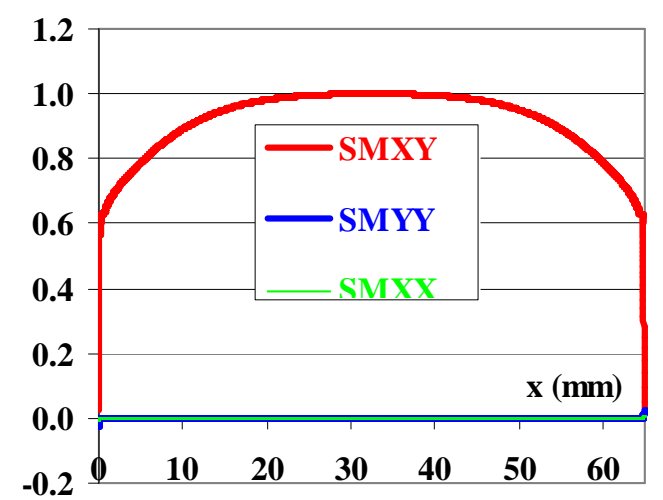

(a) stress distribution in the mean plane of the adhesive

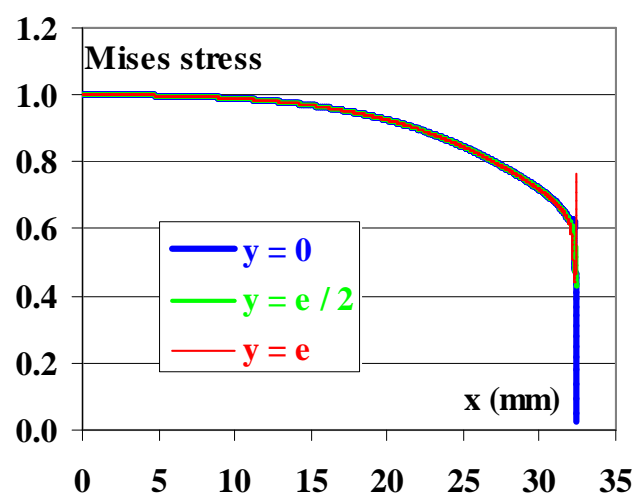

(b) results for geometry "A"

Fig. 5. Normalized stress through the thickness of the joint in the case of steel substrates.

Several analytical studies have been proposed to analyze the influence of the geometry on the stress singularities for bi-material joints [13-15]. In the case of elastic behavior the two main parameters are the relative elastic properties of the material (adhesive and substrate) and the geometries close to the edges (both the substrates and the edges of the adhesive layer). Those studies show that the use of sharp beaks is useful in designing a bi-material assembly with low stress singularities, but with those approaches it is difficult to analyze the influence of the numerous parameters on the stress distribution in the joint. For instance, the geometry of the bonded assembly and the external loading on the structure can modify the stresses within the adhesive joint and thus modify the stress singularities. Moreover as the thickness of the adhesive layer is very small with respect to the dimensions of the structure, interactions between the two interfaces can exist. Precise finite element computations are therefore useful to analyze the stress singularities in order to optimize the design of adhesive bonded assemblies.

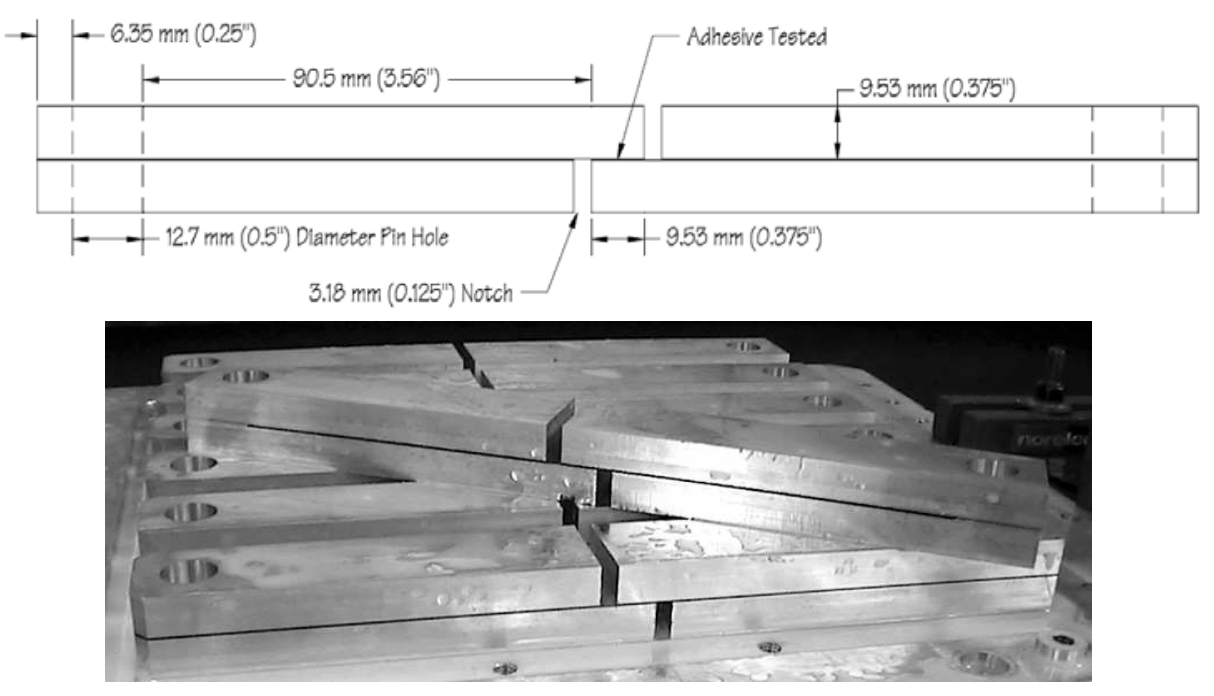

(a) geometry of the TAST specimen

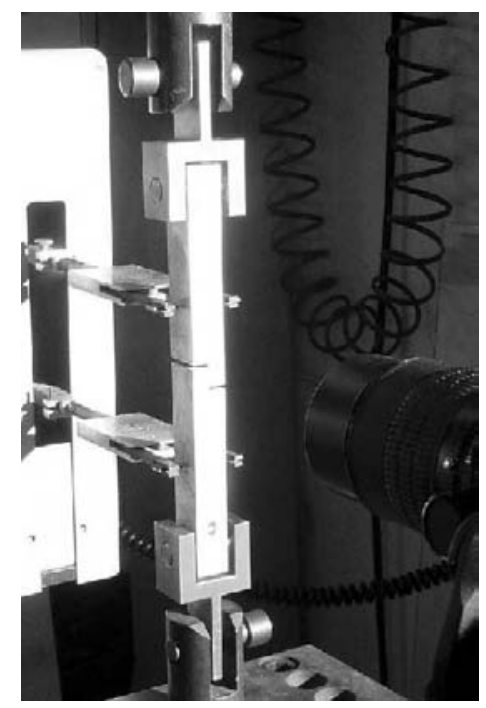

(b) test set-up

Fig. 6. Presentation of the TAST specimen (width: $25.4 \mathrm{~mm}$ ).

\section{Presentation of the TAST fixture}

The thick adherend shear test is a logical extension of the single lap shear (SLS) test method which is widely-used to evaluate adhesive systems. It was developed in Germany and the USA in the 1970's. Using thick adherends, typically $10 \mathrm{~mm}$ thick, Figure 6a, and a short overlap enables the peel stresses, which complicate the SLS, to be significantly reduced. The test is detailed in an ASTM standard and with a suitable extensometer allows the full shear stress-shear strain curve to be obtained [7]. Therefore this fixture is often used to analyze the mechanical behavior of adhesives 
in bonded assemblies [8; 16-18]. This section first presents some experimental results with adhesives showing a range of behaviors, in order to examine failure modes. Then, in order to analyze the experimental results, a numerical analysis of the stress distribution under the assumption of linear elastic behavior of the components is proposed.

\subsection{Experimental results}

The same non-contact extensometry system as used for Arcan test displacement measurements [6], based on image correlation [19], was used to analyze the kinematics of the bonded joint deformation for the TAST test. A standard extensometer was also used to verify the relative displacement of the substrates (figure 6b).

A series of preliminary tests was performed with steel substrates and three different adhesives A, $\mathrm{B}$ and $\mathrm{C}$, with $1 \mathrm{~mm}$ bondline thickness (thicker than the other tests described here) but with very different behavior. $\mathrm{A}$ is a brittle high temperature epoxy adhesive, $\mathrm{B}$ is a more ductile epoxy and $\mathrm{C}$ is a very ductile acrylic. Figure 7a presents the experimental results (DT the relative displacement of both ends of the adhesive joint and FT the tangential component of the applied load). Adhesive A shows a very small deformation before fracture, therefore the analysis of the image gives little information about the kinematics of the adhesive deformation (images of 1280 pixels x 1024 pixels are used here, a higher definition camera would provide more data). For the two other adhesives one can observe some aspects of the kinematics of the adhesive deformation. For this test the crosshead displacement rate of the tensile testing machine is $0.5 \mathrm{~mm} / \mathrm{min}$ and an image is recorded every second. Figures 7c-d present the deformation of the adhesives B and C measured on the edge of the useful zone of the TAST specimen (Figure 7b) before loading and just before failure; lines have been added to mark the interfaces adhesive-substrate. One can notice that cracks appear on the edge of the adhesive joint at the interface adhesive-substrate which can modify the analysis of the experimental results. In fact as there are 4 corners it is not easy to determine the starting time of the first crack (cameras would need to be placed on both sides of the TAST specimen). Further results are presented in the following section.

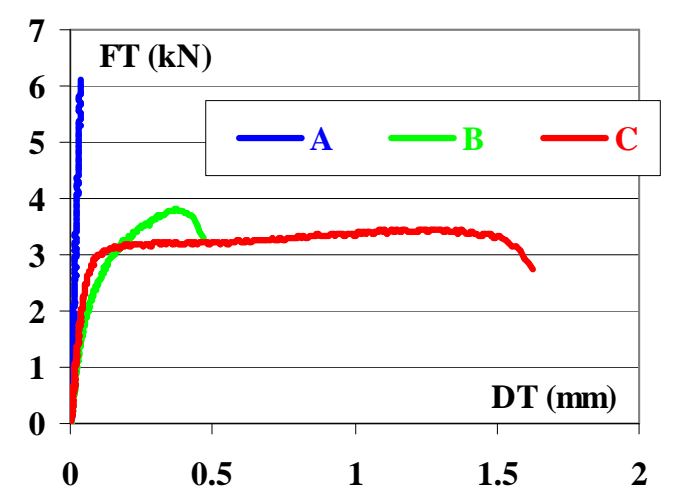

(a) experimental results

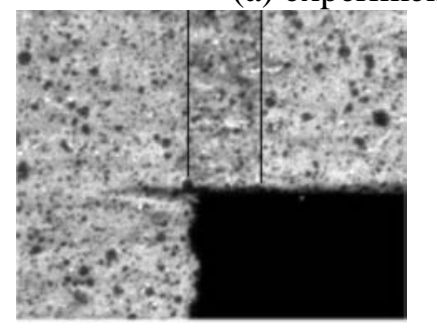

initial image

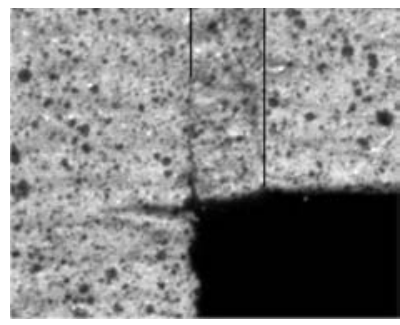

joint before failure

(c) adhesive B

(c) adhesive B
Fig. 7. Experimental results for 3 adhesives with steel substrates.

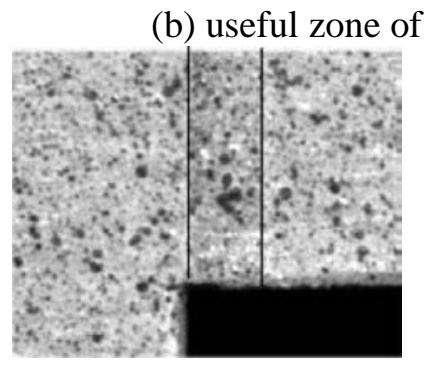

initial image

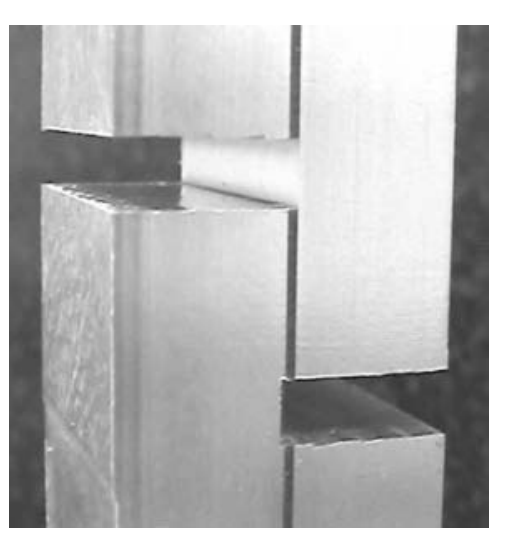

(b) useful zone of TAST specimen

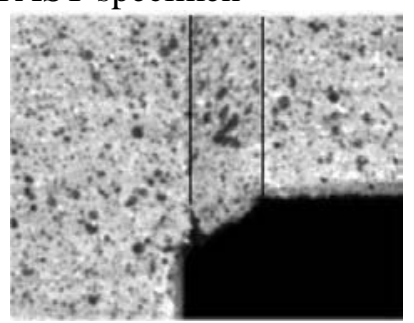

joint before failure

(d) adhesive $\mathrm{C}$
steel substrates. 


\subsection{Numerical analysis}

In order to understand the mechanics of the TAST method a 2-D FE analysis was performed, modelling half the specimen by using adequate (anti-symmetric) boundary conditions in the average plane of the adhesive joint (segment [X, Y], fig. 8). As for the Arcan test, a mesh with 40 linear rectangular elements in half of the thickness of the adhesive $(\mathrm{e}=0.2 \mathrm{~mm})$ was used. An imposed displacement on segment $[\mathrm{U}, \mathrm{V}]$ represents the loading. Numerical results are presented for the useful part of the adhesive (central part) and for the adhesive between the two substrates ("Adhesive (support)", "non-useful" part, fig 8). Figure 9 presents the distribution of the stresses in the mean plane of the adhesive joint (useful part) under the assumption of linear elastic behavior for the various components (aluminium substrates: $\mathrm{E}=80 \mathrm{GPa}, \mathrm{v}=0.3$; steel substrates: $\mathrm{E}=210 \mathrm{GPa}, \mathrm{v}=0.3$; adhesive: $\mathrm{E}=2.2 \mathrm{GPa}, v=0.3$ ). In figure 9 the shear stress is identified as "SMXY" and is normalized to unity in the middle of the joint. The use of steel substrates reduces the stresses close to the free edges, figure $9-\mathrm{a}$, in the middle plane of the adhesive, compared to aluminium substrates, figure $9 \mathrm{~b}$.

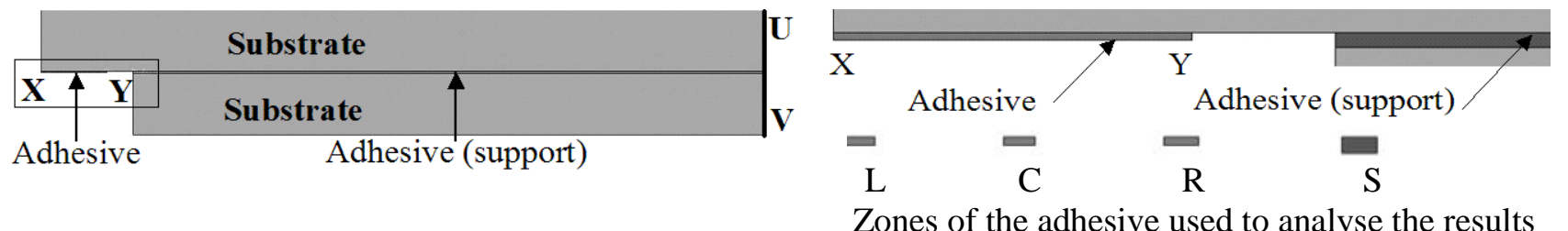

(a) geometry used

Zones of the adhesive used to analyse the results

(b) zoom

Fig. 8. Model used for the TAST test

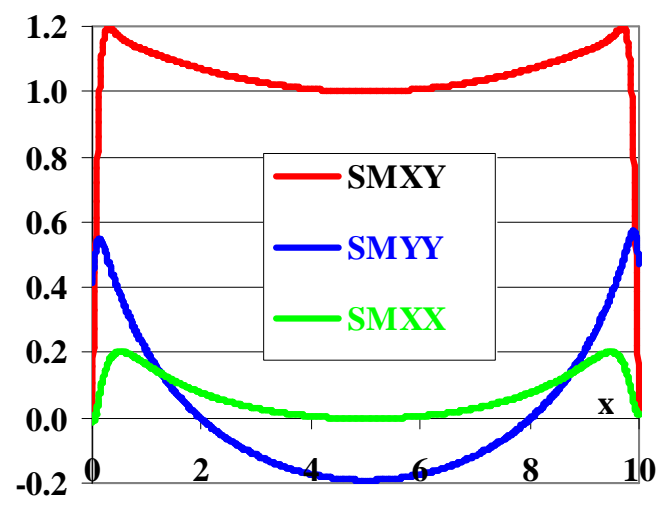

(a) aluminium substrates

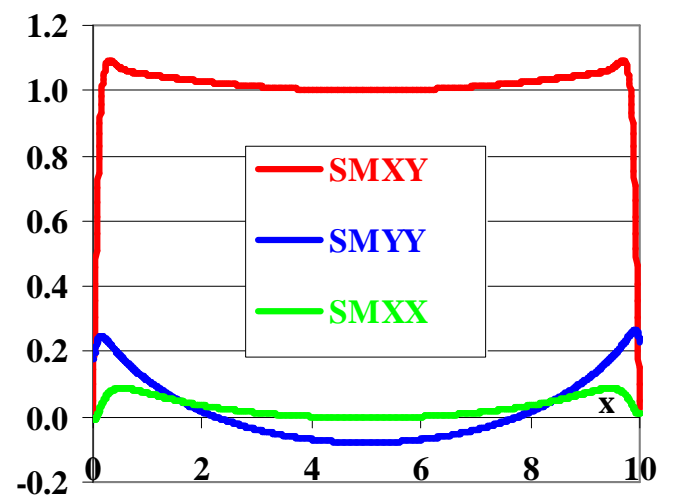

(b) steel substrates

Fig. 9. Normalized stress distribution in the middle plane of the adhesive, TAST.

These results show differences between the TAST and Arcan specimens under shear loading, especially near the edges of the adhesive joint. Near the edges, quite large normal stresses are noted for the TAST assembly, so the loading of the adhesive is not only shear. The rigidity of the connecting parts between the useful (central) part of the TAST specimen and the tensile machine depends on the material of the substrates, which can explain the influence of the substrate material on the stress distribution in the middle plane of the adhesive joint.

Figure 10 shows the large edge effects in the adhesive (in the useful zone) at the ends of the interface adhesive-substrate. The different curves correspond to three positions in the adhesive joint; $y=0$ represents the mid plane of the adhesive joint, $y=e$ is at the adhesive/substrate interface. Moreover, it is interesting to note that there are also significant edge effects in the zone noted " $\mathrm{S}$ " in figure 8 ("non-useful" zone). Those local effects in the adhesive in zone "S" have little influence on the stress distribution in the useful part of the adhesive. As underlined previously, for the TAST, the edge effects are more important for aluminium substrates than for steel ones (figures 10 and 11) whereas for the Arcan test it is the opposite. 


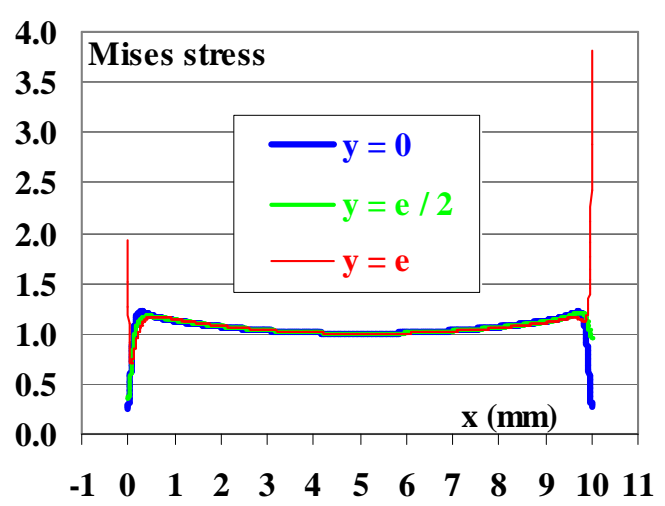

(a) edge effects in the useful part of joint

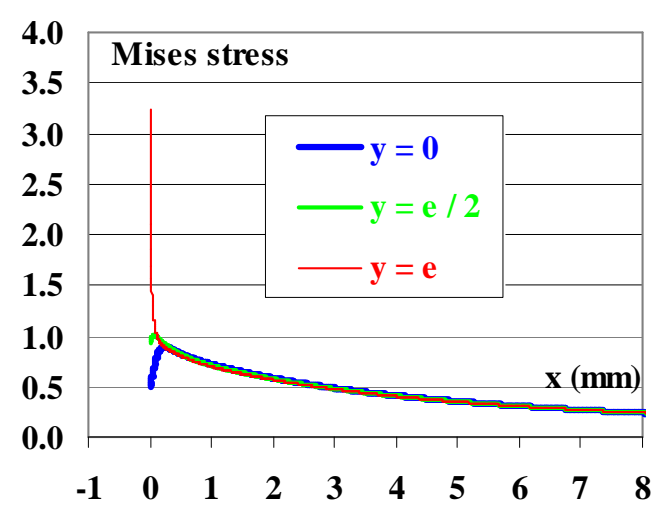

(b) edge effects on the joint in the support ess for aluminium substrates, TAST.

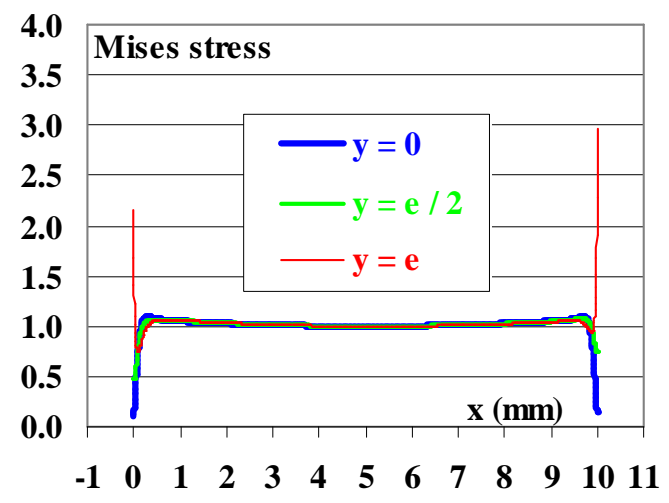

(a) edge effects in the useful joint

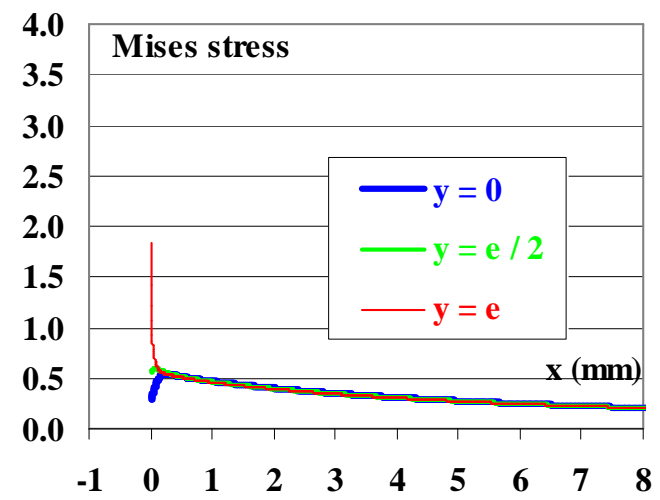

(b) edge effects on the joint in the support

Fig. 11. Normalized stress through the thickness for steel substrates, TAST.

\section{Arcan-TAST Comparison}

The two previous sections indicate some characteristics of the Arcan and TAST tests under shear loading. In this section, results from the two tests are presented for the same aluminium alloy (2017) substrates bonded with Redux 420 epoxy adhesive with the same surface preparation (abrasion with 120 grade paper similar to that used in a boatyard environment), manufactured at the same time following the same cure cycle $\left(12 \mathrm{~h}\right.$ ambient temperature, $\left.4 \mathrm{~h} 50^{\circ} \mathrm{C}\right)$, and with the same bondline thicknesses (all in the range $0.5-0.65 \mathrm{~mm}$ ) in order to complete the comparison.

\subsection{Experimental results}

Figures 12 and 13 present the experimental results, for two imposed speeds of the displacement of the crosshead of the tensile testing machine. For these tests, corresponding to shear loading of the adhesive, the evolution of the effort transmitted by the joint (denoted by FT) with respect to the relative displacement of the two ends of the adhesive joint (denoted by DT) is plotted for displacement rates of the crosshead of the tensile testing machine of 0.5 and $10 \mathrm{~mm} / \mathrm{min}$. Figures 12 and 13 also show images of the evolution of the deformation of the adhesive joints for different points indicated in the load-displacement plot, corresponding to tests at a loading rate of $0.5 \mathrm{~mm} / \mathrm{min}$. Moreover, for a loading rate of $0.5 \mathrm{~mm} / \mathrm{min}$, an idea of the scatter in the experimental results is presented in figure 12-b and figure 13-b. Those figures show results from tests on four specimens and indicate that test variability is quite low.

It is important to note that the cross-sections Sc of the adhesive plane are different for the two tests: $\mathrm{Sc}=9.53 \times 25.4 \mathrm{~mm}^{2}$ for the TAST while $\mathrm{Sc}=10 \times 65 \mathrm{~mm}^{2}$ for the Arcan test. A comparison of the experimental results in shear for these two tests shows differences in the non-linear behavior. For the Arcan test a "homogeneous" deformation of the adhesive joint is observed and a rising loaddisplacement plot after the knee. On the other hand, for the TAST, cracks appear quickly at the two 
edges of the adhesive joint close to the adhesive-substrate interface. Those cracks modify the resistant area of the assembly and thus can explain the decrease in the load transmitted by the bonded joint after a given DT. In fact the specimen is a structure with crack initiation near the substrate-adhesive interface, followed by crack propagation.

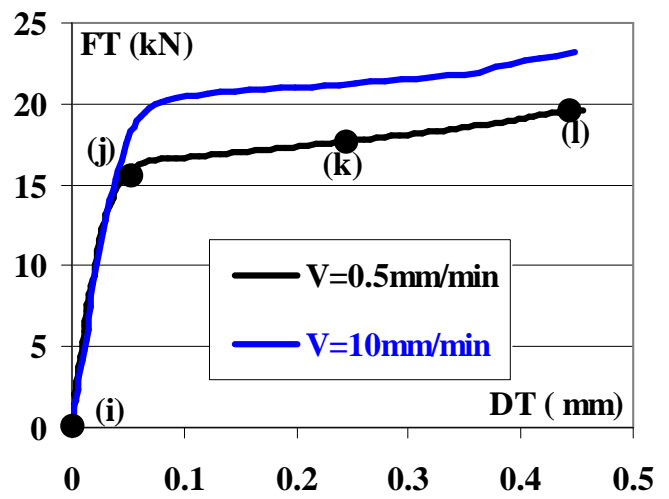

(a) experimental results for two loading rates

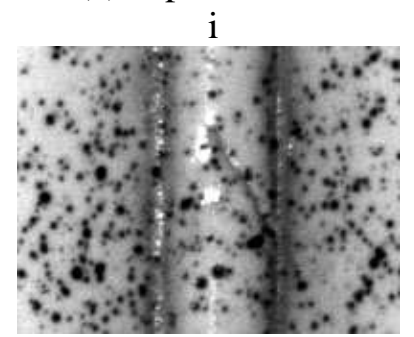

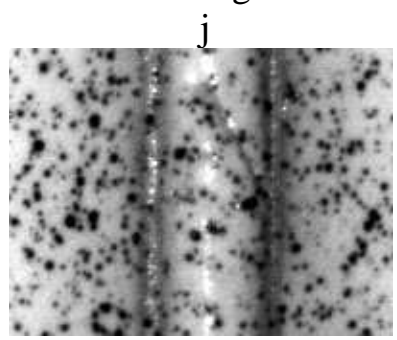

(c) evolution of the deformation of the adhesive joint

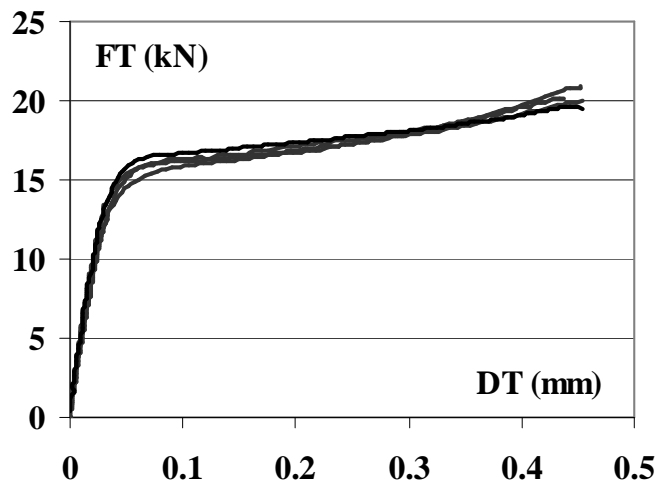

(b) results from 4 tests $(\mathrm{V}=0.5 \mathrm{~mm} / \mathrm{min})$
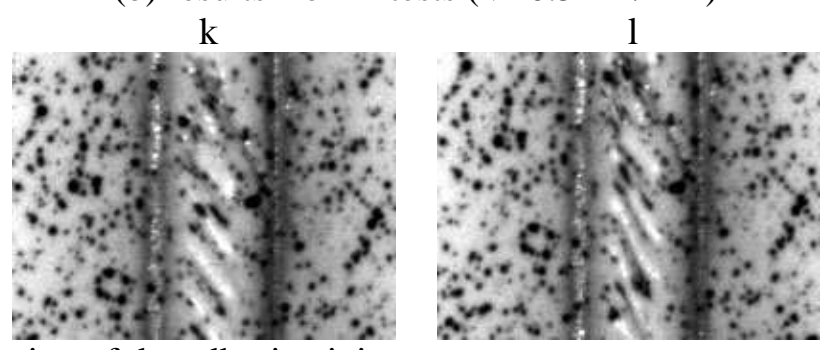

Fig. 12. Arcan test, joint thickness: $0.5 \mathrm{~mm}$.

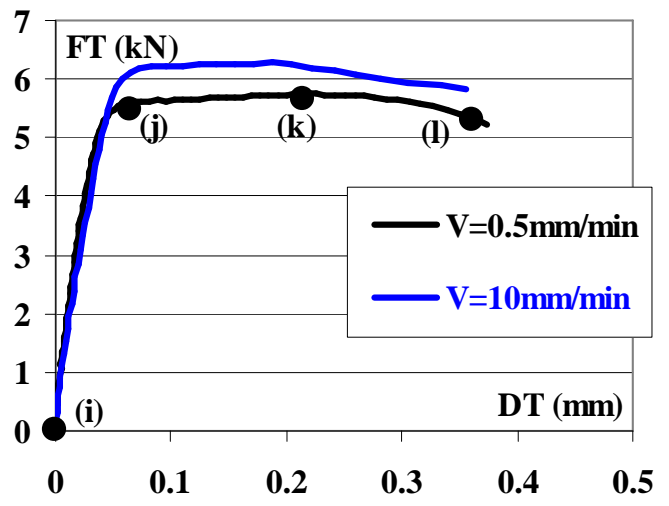

(a) experimental results for two loading rate

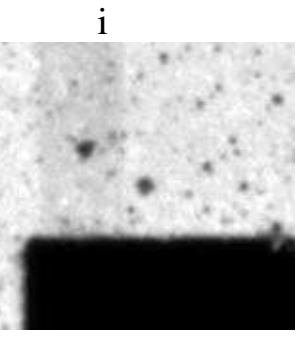

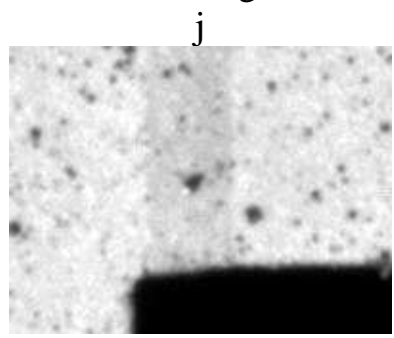

(c) evolution of the deformation of the adhesive joint

Fig. 13. TAST test, joint thickness: $0.55-0.65 \mathrm{~mm}$.

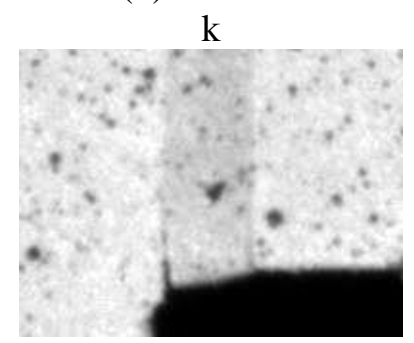

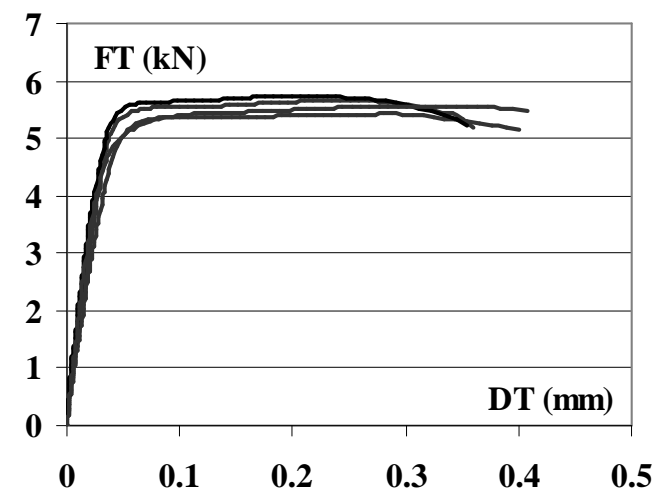

(b) results from 4 tests $(\mathrm{V}=0.5 \mathrm{~mm} / \mathrm{min})$

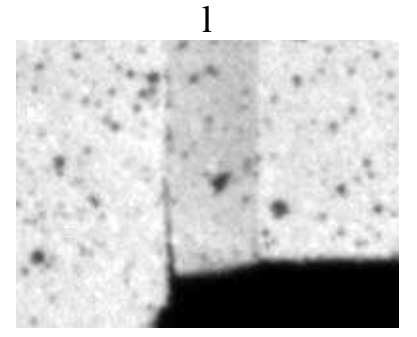

\subsection{Analysis of the experimental results}

As the numerical simulations (performed using linear behavior of the constituents) have shown a non uniform evolution of the state of stress in the adhesive joint, an inverse technique has been used 
to identify the parameters of the material behavior model for the Arcan test [6]. For monotonic loadings, elasto-plastic behavior with isotropic hardening allowed the experimental results to be accurately represented.

For the TAST, ASTM D5656-95 defines a "limit" of elasticity based on the change of slope of the diagram of average stress FT/Sc versus relative displacement DT; Sc is the section of the adhesive plane. As the stress distribution in the mid plane of the adhesive is not uniform, the maximum shear stress, for aluminium substrates, is close to $1.14 * \mathrm{FT} / \mathrm{Sc}$ (using the material properties identified for the adhesive using the Arcan test). This value is obtained using the results presented in figure 9. In fact, knowing the stress distribution in the mid-plane of the adhesive, one can obtain the transmitted force. Thus, the average shear stress can be compared to the maximum shear stress $\left(\sigma_{\mathrm{xy} \operatorname{maxi}}=1.14 \sigma_{\mathrm{xy}}\right.$ average, with $\sigma_{\mathrm{xy}}$ average $\left.=\mathrm{FT} / \mathrm{Sc}\right)$. A 3-D calculation gives similar results. This procedure, inspired by the inverse identification technique developed for the Arcan test, makes it possible to obtain a slightly higher value for this elastic limit. The value denoted by "rupture" is associated with the ultimate load. Figure 14 presents a comparison of the analyses of the experimental results from the two tests for a crosshead displacement rate of the tensile testing machine of $0.5 \mathrm{~mm} / \mathrm{min}$.

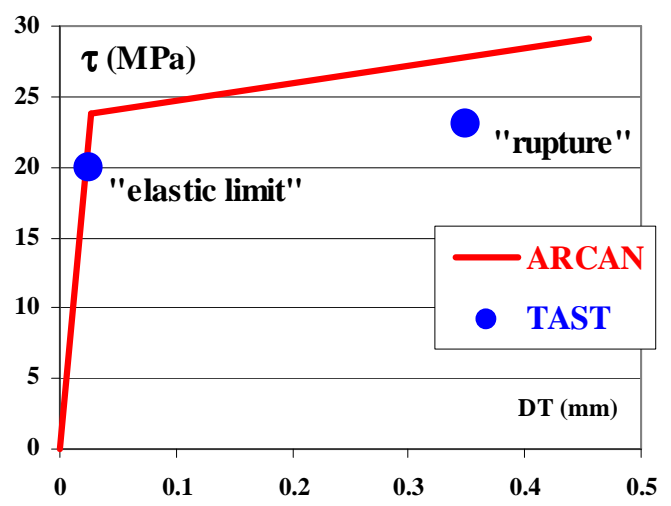

Fig. 14. Identified behavior for the adhesive joint under shear loading $(0.5 \mathrm{~mm} / \mathrm{min})$.

\subsection{Influence of the non-linear behavior of the adhesive}

As the Redux 420 adhesive joint shows strongly non-linear behavior, in particular under shear loads, it is interesting to take this into account in the analysis of these tests. Simulations were carried out under the assumptions of finite transformations and elastic-plastic behavior of the adhesive with a Von Mises criterion, identified based on the Arcan tests under shear loading (fig. 14). For these applications, where the shear stress component in the adhesive dominates, the use of an elasto-plastic model with isotropic hardening is acceptable. The results are shown in Figures 15 and 16 for the Arcan and the TAST specimens, respectively.

In order to facilitate the analysis of the numerical results, figures 15 and 16 present them for the zoomed area of the adhesive shown in figure 8 for TAST specimen. For the TAST results are shown for the left (L), the central (C), and the right (R) parts of the joint, together with the point labelled $S$ (not in the useful part of the adhesive). For the Arcan test the same three parts are used: L, C and R. Figures 15 and 16 present, for relative displacements of the ends of the joint of DT $=0.03 \mathrm{~mm}$ and of DT $=0.06 \mathrm{~mm}$ and a thickness of the joint of adhesive of $0.4 \mathrm{~mm}$, the state of the cumulated plastic deformation; for each zone the extreme values are noted above the figures. The TAST test is characterized by the development of strongly plasticized zones at the edges of the adhesive joint close to the interface (a "zoom" of the scale makes it possible to visualize the phenomenon more easily). For the two tests, the central parts of the adhesive joint are loaded in a similar way. The nonlinear geometric effects may explain the differences between zones L and R. Figures 15-c and 16-c present the evolution of the maximum value of the cumulated plastic deformation in the different zones with respect to DT. Moreover, for the Arcan test, the solutions are also plotted for the elements of the adhesive joint located at the two edges of the interface adhesive-substrates 
(references $\mathrm{r}$ and 1 ). For those numerical simulations, crack initiation and crack propagation are not taken into account, thus the real behavior (load decreasing with crack development) of the TAST can not be shown. However, the important point is to underline the high level of cumulated plastic deformation at the edge of the useful part of the adhesive (and, to a lesser extent, in the part noted "S") which can explain crack initiations.

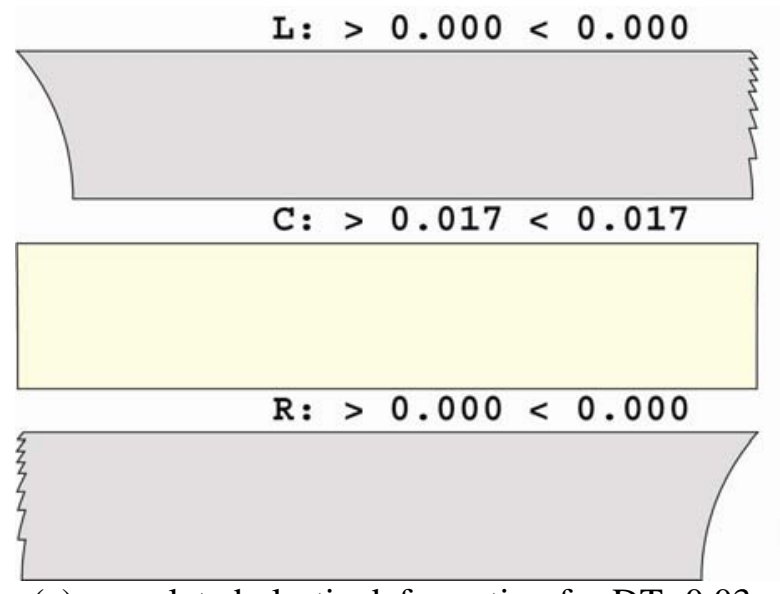

(a) cumulated plastic deformation for DT $=0.03 \mathrm{~mm}$

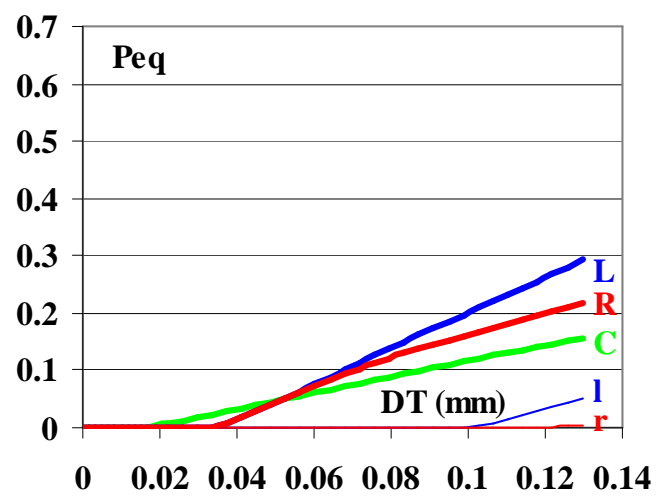

(c) Evolution of cumulated plasticity

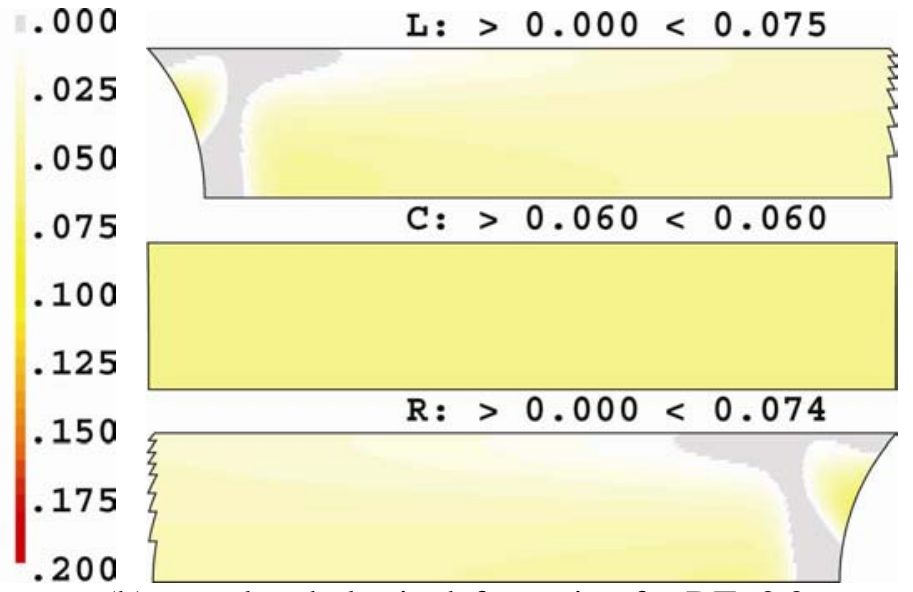

(b) cumulated plastic deformation for DT $=0.06 \mathrm{~mm}$

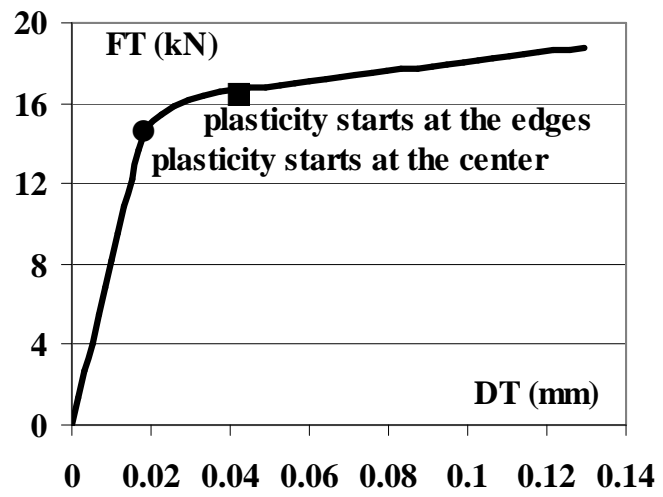

(d) Load-Displacement diagram

Fig. 15. Results of the non-linear simulation for the Arcan test.

This numerical study underlines the differences between these two tests. For the TAST, the zones close to the interfaces are the most highly stressed regions of the joint. Therefore, cracks can appear in that area. On the other hand, for the modified Arcan test, the adhesive joint is stressed in a much more homogeneous way. Moreover, the maximum stress (close to the centre) is obtained close to the free edge of the adhesive but not near the interface. It is important to note that the interface adhesive-substrate is often the weakest part of the assembly either due to defects on the surface or to local variations in chemistry [20]. Thus the Arcan test makes it possible to obtain larger relative displacements DT than the TAST test. It is important to underline however, that these are numerical simulations and can only provide indicative results. Nevertheless, Figures $15-\mathrm{d}$ and 16-d present the load-displacement diagrams for the two tests and the results obtained are similar to the experimental data. These figures also present the point associated with the beginning of plasticity within the central zone and at the edges of the adhesive joint, and indicate the early appearance of plasticity in the TAST specimen. 


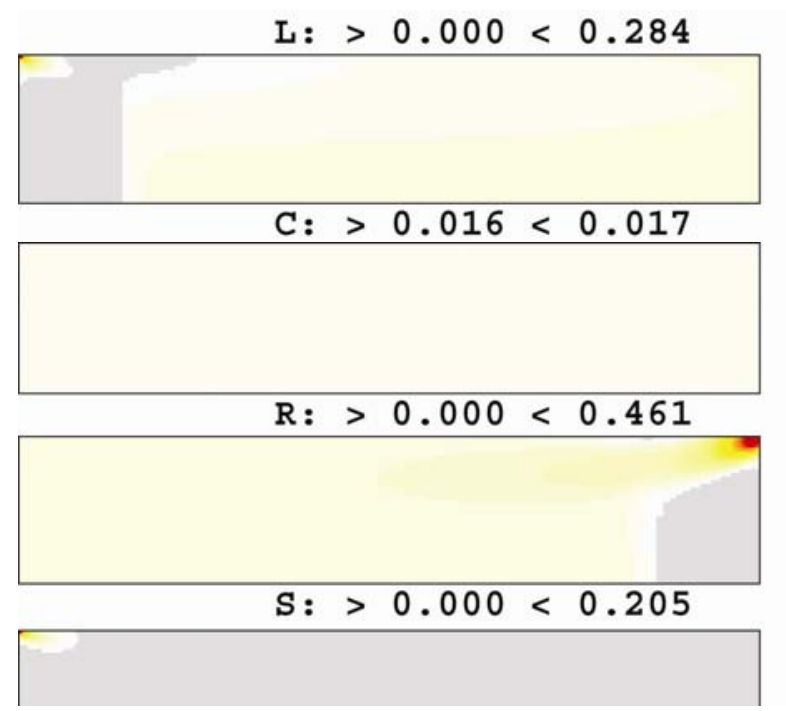

(a) cumulated plastic deformation for DT $=0.03 \mathrm{~mm}$

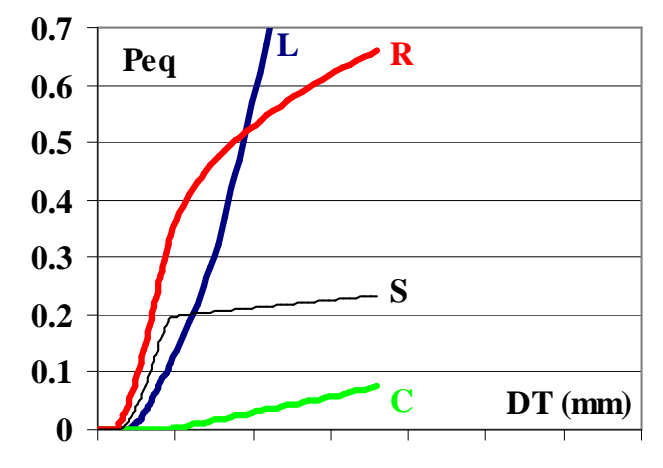

$\begin{array}{lllllllll}0 & 0.02 & 0.04 & 0.06 & 0.08 & 0.1 & 0.12 & 0.14\end{array}$

(c) Evolution of cumulated plasticity

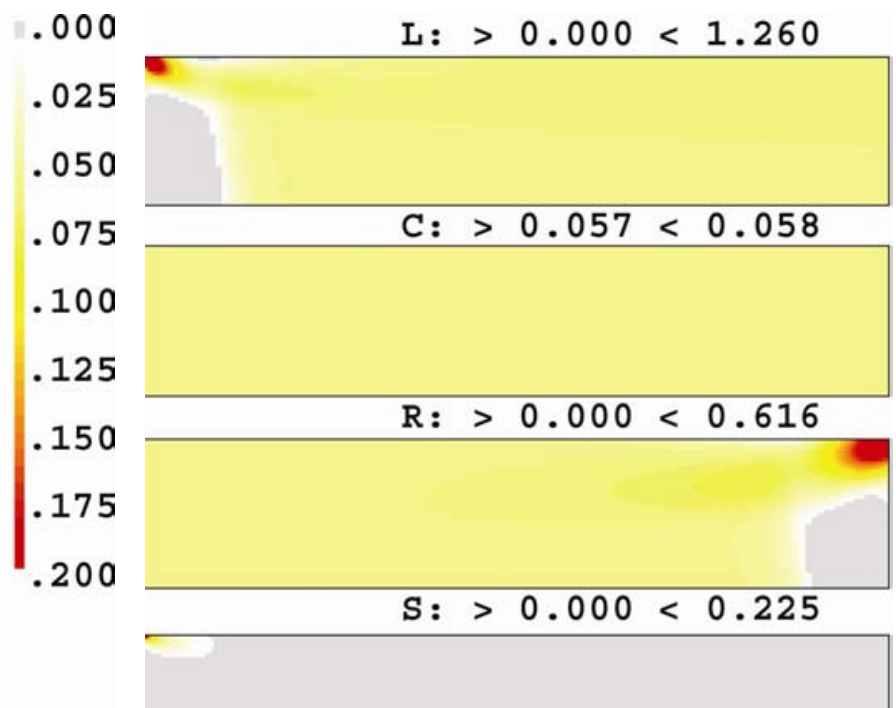

(b) cumulated plastic deformation for DT $=0.06 \mathrm{~mm}$

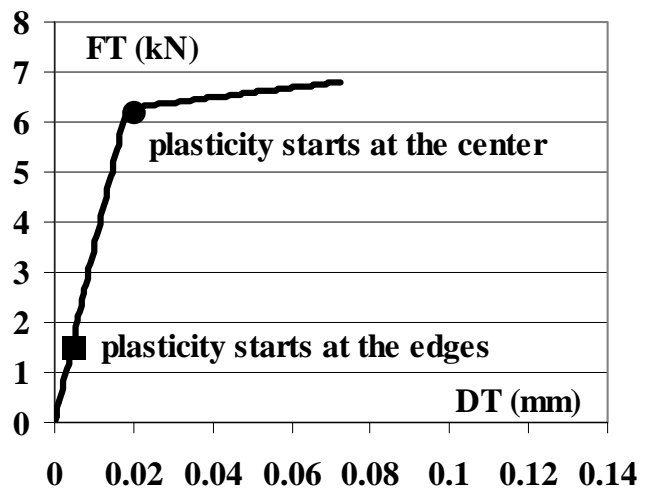

(d) Load-Displacement diagram

Fig. 16. Results of the non-linear simulation for the TAST.

\section{Proposal of an improved TAST test}

The principal difference between the two tests is associated with the edge effects. It is thus natural to study the influence of the use of beaks in order to limit these effects in the TAST. From a practical point of view the machining of the beaks first requires the machining of grooves much larger on both sides of the useful part of the joint, as shown in Figure 17.
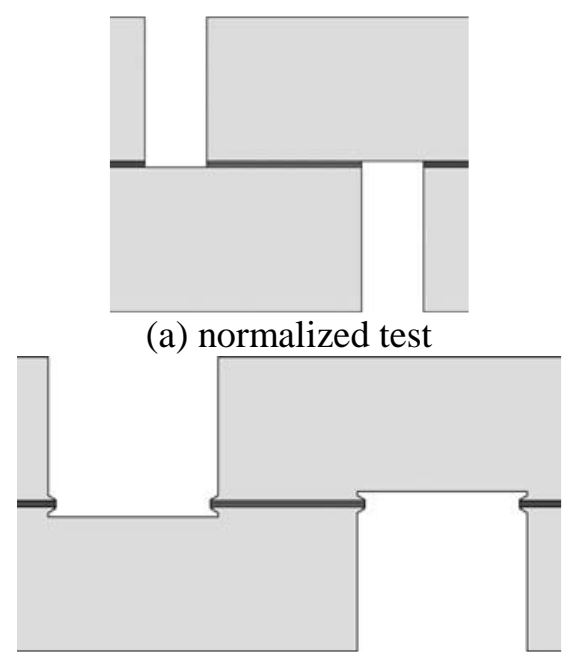

(c) machining of beaks

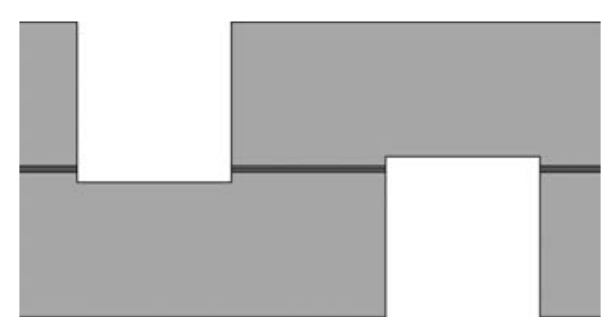

(b) machining of deeper grooves

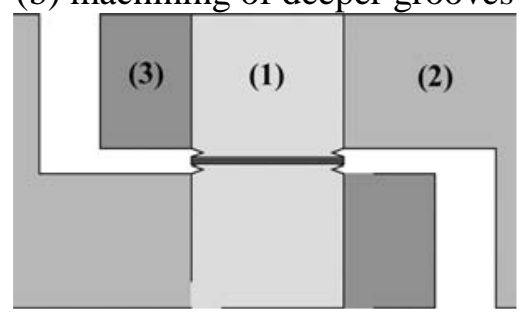

(d) modified test

Fig. 17. Proposed modifications to TAST geometry to reduce edge effects. 


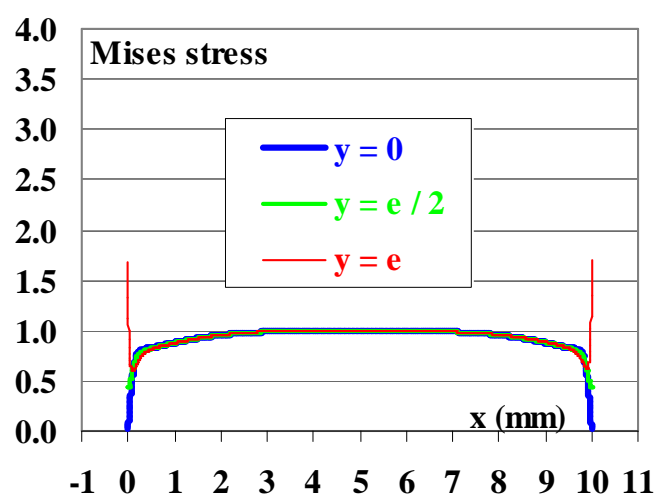

(a) edge effects in the useful joint

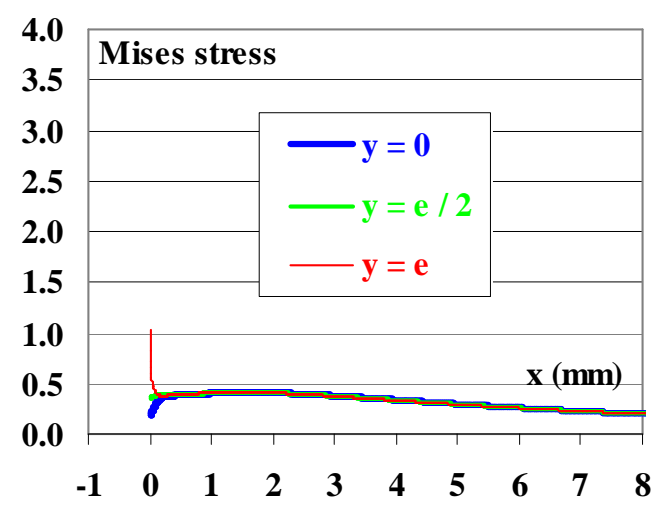

(b) edge effects on the joint in the support

Fig. 18. Normalized stress through the thickness for aluminium substrates with deeper grooves.

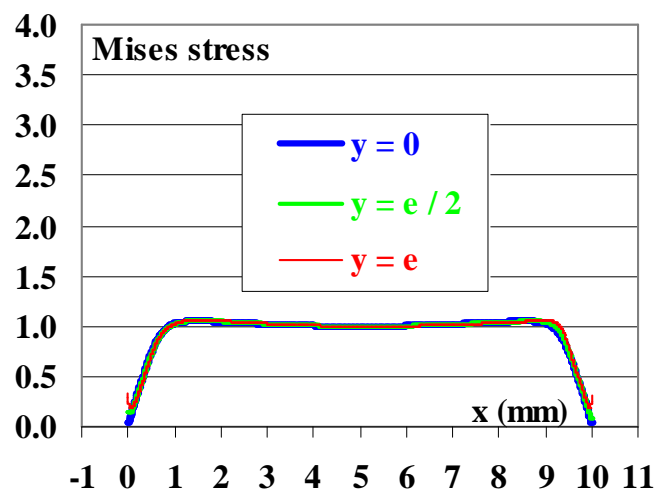

(a) edge effects in the useful joint

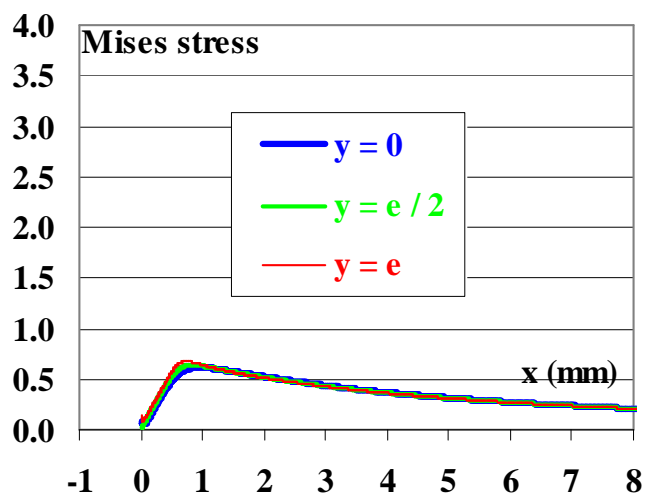

(b) edge effects on the joint in the support

Fig. 19. Normalized stress through the thickness for aluminium substrates with sharp beaks.
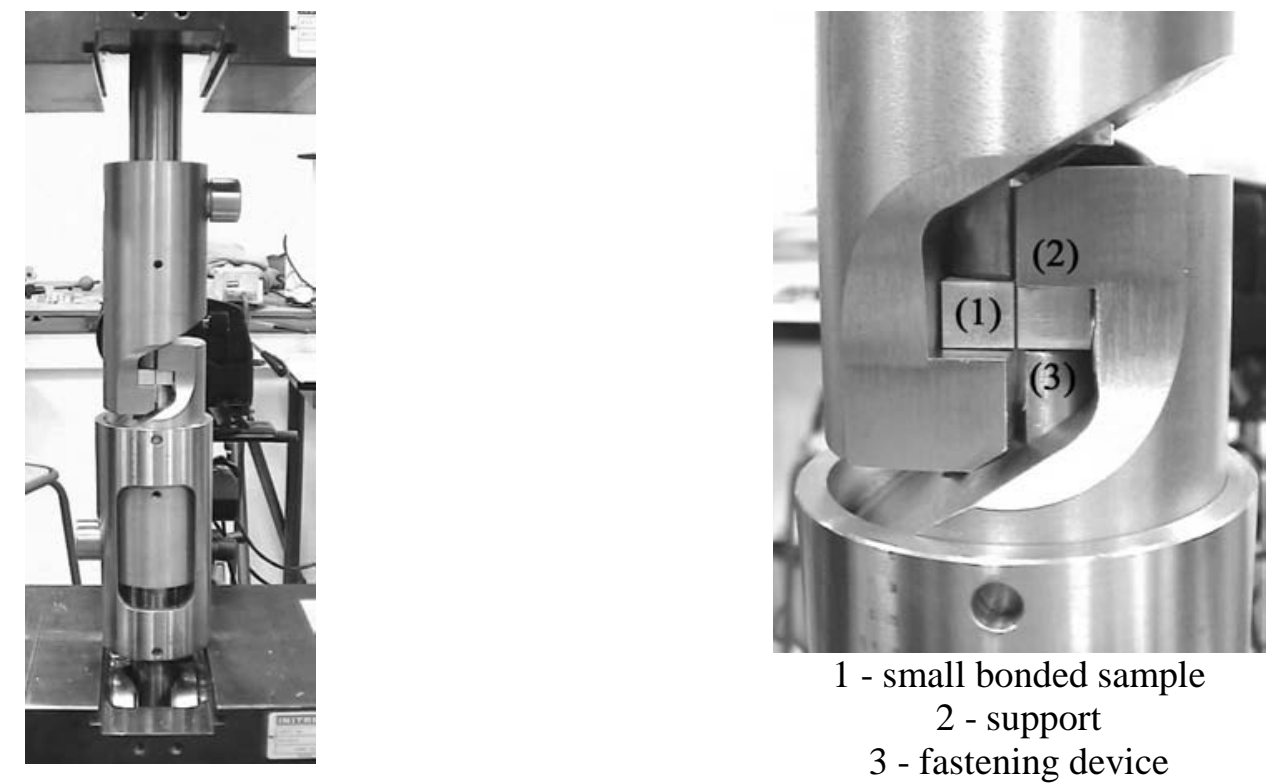

Fig. 20. Prototype modified TAST fixture.

The numerical analysis of these modifications of the geometry of the substrates close to the useful part of the joint makes it possible to examine how they affect the stress state. First, it is important to note that simply machining deeper groves changes the stress distribution in the adhesive joint and limits the edge effects with respect to the normalized TAST approach, as shown in figures $17 \mathrm{~b}$ and 18 . Those results should be compared with the results for the normalized TAST 
presented in figure 10 with the same scale. Then, adding sharp beaks can strongly reduce the edge effects (figures 17c and 19).

Finally, as the machining of the beaks within the TAST specimen is not an easy operation, a second possibility is to use a modular assembly with small bonded samples (noted (1) in Figure 17d) which are tested in a re-usable support fixture. The samples can be cut out of the bonded plates used for the machining of normalized TAST samples (parallelepiped height approximately $20 \mathrm{~mm}$ with an adhesive joint of $\mathrm{Sc}=9.53 \times 25.4 \mathrm{~mm}^{2}$ section in the mid-plane). The beaks are then much easier to machine. They are mounted in a support (noted (2) in fig. 17-d) and fixed with a fastening device ((3) in fig. 17-d). The first results, obtained using a prototype device (Figure 20) seem to be promising. Moreover, this device is well suited to the study of the influence of the ageing of adhesives, as it uses small samples, which are much less expensive than aging complete TAST specimens. The optimization of the geometry of these modified TAST samples, which require high stiffness in bending in order to limit parasitic effects, is currently in progress.

\section{Conclusion}

This paper presents results from a study to optimize tests to characterize the mechanical behavior of adhesive joints for marine structures. In order to validate results from a modified Arcan fixture, which uses a beak to reduce edge effects, shear properties were measured using the standard TAST configuration. The test results indicate differences in the measured shear behavior, the TAST specimen giving a different non-linear behavior and damage initiates near the adhesive/substrate interface rather than within the adhesive.

A numerical study has shown that for the TAST fixture the edge effects are very significant and explain some of the differences between the two tests. Moreover it has been shown that the two parameters usually examined, the relative elastic properties of the material (adhesive and substrate) and the geometries close to the edges (both the substrates and the edges of the adhesive layer), are not sufficient to analyze the edge effects. In order to obtain precise results, it is necessary to take into account the influence of the fixture: the rigidity of the different parts of the bonded assembly and the external loading on the structure can modify the stresses within the adhesive joint and can thus modify the stress singularities. For instance, in the case of the Arcan test the edge effects are larger for steel substrates than for aluminium, whereas for the TAST the opposite is noted. For the TAST with both substrates, the zones close to the interfaces are the most highly stressed parts of the adhesive joint. Therefore, cracks can appear near them and they can have an influence on the experimental results (i.e. the load versus relative displacements of both ends of the adhesive joint). On the other hand, for the Arcan test, the adhesive joint is stressed in a much more homogeneous way. Thus, the modified Arcan test makes it possible to obtain more reliable experimental data than the TAST, particularly when an adhesive failure mode is dominating.

The experience gained during improvement of the design of the Arcan assembly have made it possible to propose modifications to the TAST fixture, to give a more homogeneous adhesive stress state. A modular assembly with small samples with machined beaks mounted in a re-usable support fixture is proposed, which may provide a low cost solution to aging studies of adhesive assemblies. Tests are underway to complete these studies.

\section{Acknowledgements}

The authors acknowledge the Bretagne Region of France for supporting this work.

\section{References}

[1] Adams RD. Adhesive bonding: Science, technology and applications, Woodhead Publishing Limited; 2005.

[2] Tong L. Steven GP. Analysis and design of structural bonded joints, Kluwer; 1999.

[3] Arcan L, Arcan M, Daniel L. SEM fractography of pure and mixed mode interlaminar fracture in graphite/epoxy composites, ASTM Tech Publ 1987; 948: 41-67. 
[4] Cognard JY, Davies P, Gineste B, Sohier L. Development of an improved adhesive test method for composite assembly design, Composite Science \&Technology 2005; 65: 359-68.

[5] Cognard JY, Créac'hcadec R, Davies P, Sohier L. Numerical modelling of the non-linear behavior of adhesively-bonded assemblies, in "Innovation In Engineering Computational Structures Technology", Saxe-Coburg Publications, ISBN 1-874672-27-X 2006, Chapter 11, 225-47.

[6] Cognard JY, Davies P, Sohier L, Créac'hcadec R. A study of the non-linear behavior of adhesively-bonded composite assemblies, Composite Structures 2006; 76: 34-46.

[7] ASTM D5656-95. Standard Test Method for Thick-Adherend Metal Lap-Shear Joints for Determination of the Stress-Strain Behavior of Adhesives in Shear by Tension Loading, ASTM 1995.

[8] Tomblin J, Seneviratne W, Escobar P, Yoon-Khian Y. Shear Stress-Strain data for structural adhesives, DOT/FAA/AR-02/97 report, 2002.

[9] Wu Z. Design free of stress singularities for bi-material components, Composite Structures 2004; 65: 339-45.

[10] Da Silva LFM, Adams RD. Techniques to reduce the peel stresses in adhesive joints with composites, Int J Adhesion Adhesives 2007; 27: 227-35.

[11] Cheikh M, Coorevits P, Loredo M. Modelling the stress vector continuity at the interface of bonded joints, Int J Adhesion Adhesives 2001; 22: 249-57.

[12] Magalhães AG, De Moura MFSF, Goncalves JPM. Evaluation of stress concentration effects in single-lap bonded joints of laminate composite material, Int J Adhesion Adhesives, 2005; 25: 313-9.

[13] Leguillon D, Sanchez-Palancia E. Computation of singular solutions in elliptic problems and elasticity, Editions Masson, Paris; 1987.

[14] Kotousov A., "Effect of a thin plastic adhesive layer on the stress singularity in a bi-material wedge", Inter J Adhesion Adhesives 2007; 27: 647-52.

[15] Wang P, Xu LR. Convex interfacial joints with least stress singularities in dissimilar material, Mechanics of Materials 2006; 38: 1001-11.

[16] Brinson HF. The viscoelastic constitutive modeling of adhesives, Composites 1982: 377-82.

[17] Chiu WK, Chalkley PD, Jones R. Effects of temperature on the shear stress-strain behavior of structural adhesive, Computers Structures 1994; 53: 483-89.

[18] Zgoul M, Crocombe AD. Numerical modeling of lap joints bonded with rate-dependent adhesive, Int J Adhesion Adhesives 2004; 24: 355-66.

[19] Correlated Solutions Inc., Vic2D/Vic3D, http://correlatedsolutions.com, 2002.

[20] Roche AA, Bouchet J, Bentadjine S. Formation of epoxy-diamine/metal interphases, Int J Adhesion Adhesives 2002; 22: 431-44. 